\title{
Effect of noise, order and range in fitting the photopeak region of local, Anger-camera energy spectra
}

\author{
X. Wang ${ }^{1)}$, K.F. Koral ${ }^{1)}$, N.H. Clinthorne ${ }^{1)}$, W.L. Rogers ${ }^{1)}$, C.E. Floyd Jr. ${ }^{2)}$ \\ and R.J. Jaszczak ${ }^{2)}$ \\ "Division of Nuclear Medicine, University of Michigan, Ann Arbor, MI 48109, USA \\ 2) Department of Radiology, Duke University Medical Center, Durham, NC 27710, USA
}

\begin{abstract}
In order to estimate and correct Compton scattering in nuclear-medicine Anger-camera imaging, we have previously required the least-mean-square error between the locally measured energy spectrum and one dependent on a model. The model assumes a fixed-order polynomial for the spectrum of scatter and fits the data over a specified energy range In this study, a Monte Carlo simulation program produces spectra at specified locations in a projection image of a ${ }^{99 m}$ Tc "hot" sphere in a "cold" cylinder. Poisson noise is subsequently added to each spectral channel, modelling a given count level within the acceptance window. Tests were done at two pixel locations, one at the center of the sphere and the other near the edge. Without noise, we find that the calculated-to-true ratio for unscattered counts is reasonably close to 1.0 (average 1.03 , range 0.85 to 1.16 ) for all of the 16 order-range combinations that were tested Tests on experimental data yield comparable results. For comparison, without any Compton-scatter correction the average ratio is 1.39 . Optımızing the fitting parameters is difficult because, for example, the best set for location 1 is the worst for location 2. With noisy data, the relative standard deviation, and sometimes the bias for the estimate of direct (1.e. unscattered) counts, increases as the statistical noise increases. The average relative error for the estimate $1 \mathrm{~s} 10 \%$ for the 3 cases measured with about 5000 unscattered counts but increases to $20 \%$ if that number decreases to 700 .
\end{abstract}

\section{Introduction}

Compton scattering of gamma rays creates a problem in quantitative imaging with Anger cameras in nuclear medicine, because of the finite energy resolution of the $\mathrm{NaI}(\mathrm{Tl})$ crystal. A window denoting acceptable energy avoids including gamma rays that have undergone large-angle scatters in the patient and emerge with low energies. However, with present camera energy resolution, that window cannot practically be narrowed (or offset) enough to eliminate all scattered gamma rays.

We have previously proposed a Compton scattering correction technique which does a least-square fitting between the local data energy spectrum and one dependent on a model to estimate the scatter contamination of the photopeak [1]. The model investigated to date employs a low-order polynomial for the scattered spectrum and a scatter-free energy spectral shape for the corrected (unscattered) spectrum. Validation of the calculated scatter fraction (the ratio of scattered over unscattered counts) for very good counting statistics and a third-order polynomial has been obtained experimentally [2].

In this paper, we report our latest investigation on this spectral-fitting method. First, the method is tested on Monte Carlo simulated energy spectra with almost infunitely good statistics to see the effects of fitting parameters. The results are compared with those from experimentally measured data. Then, various levels of Poisson noise are added to the good-statistics Monte Carlo energy spectra to simulate realizations with counting statistics noise, and the fitting method is applied to each to test the robustness of the method. Results in all cases are compared with the true answers provided by the Monte Carlo simulation program.

\section{Methods}

The spectral-fitting method analyzes local Angercamera spectra (those at specified locations in the image of the object), using an "energy-spectrum image". This image is a determination of the energy spectrum over a given energy range at every pixel location in the image. For each individual energy spectrum, the unscattered component is represented by a scatter-free spectrum (usually measured separately in practical situations) times a constant, and the Compton scattered component is represented by a fixed-order polynomial. The sum of the two components is then fitted to the measured spectrum to obtain both the constant and the polynomial coefficients, and thus the total counts from each component in the given energy window; see ref. [1] for more details. 
Two parameters in this method that need to be determined are the order of the polynomial and the energy-spectrum range over which the fitting process is undertaken. The higher the order, the more complicated the scatter spectrum that can be represented. But, at the same time, the fitting answer (number of direct counts) intuitively is also likely to become more sensitive to noise with an increase in the order. The range parameter, which is also called the fitting window, controls the amount of information about the measured spectrum that is being passed to the fitting algorithm. The wider this fitting window, the more scattered counts the fitting procedure can see and therefore the more accurate the fitting may be if the model is correct. However, since a more complicated scattering shape probably will be needed, a higher order will be needed and again the technique becomes more sensitive to noise.

Monte Carlo simulation of Compton scattering was studied by Floyd et al. [3]. In this study, we use that computer program to generate the energy spectra with Compton scattering. The exact phantom geometry and activity distribution is modeled by the program, which in turn calculates separate energy-spectrum images due to scattered as well as unscattered photons. The sum of the two energy spectra is used as the simulation for the measured raw data. The unscattered part alone, at the sphere center (see below), is used as the simulation for the scatter-free spectrum. A very large number of histories is employed to obtain essentially infinite-statistics spectra in most pixels. These spectra are used to test the effect of the fitting parameters (the order of the polynomial and the fitting window). A random-number generator is later used to simulate Poisson counting noise for each $2 \mathrm{keV}$ energy channel of the raw data, assuming a given mean count level. The noisy energy spectra are used to test the sensitivity of the fitting method to noise.

The phantom simulated is a nonradioactive-waterfilled cylinder with a $6 \mathrm{~cm}$ diameter sphere, containing ${ }^{99 \mathrm{~m}} \mathrm{Tc}(140 \mathrm{keV})$ in water solution, at the center of the cylinder. The cylinder has a diameter of $19.7 \mathrm{~cm}$ and a height of $25.4 \mathrm{~cm}$. A cross section of the phantom is illustrated in fig. 1. Energy spectra at two pixel locations, both inside the "hot" sphere, are used for testing. Location 1 is at the center of the sphere where the scattering is relatively low (scatter fraction $=0.245$ ). Location 2 is near the edge of the sphere where more scattering is measured (scatter fraction $=0.540$ ).

To test the fitting method, the total computed unscattered counts, also called direct counts, in a $20 \%$ symmetric energy window is compared with the true direct counts in the same energy window. For tests on the non-noisy, Monte Carlo energy spectra, the ratios of the computed direct counts over the true direct counts are calculated for each parameter combination. The percentage of the required correction which is obtained

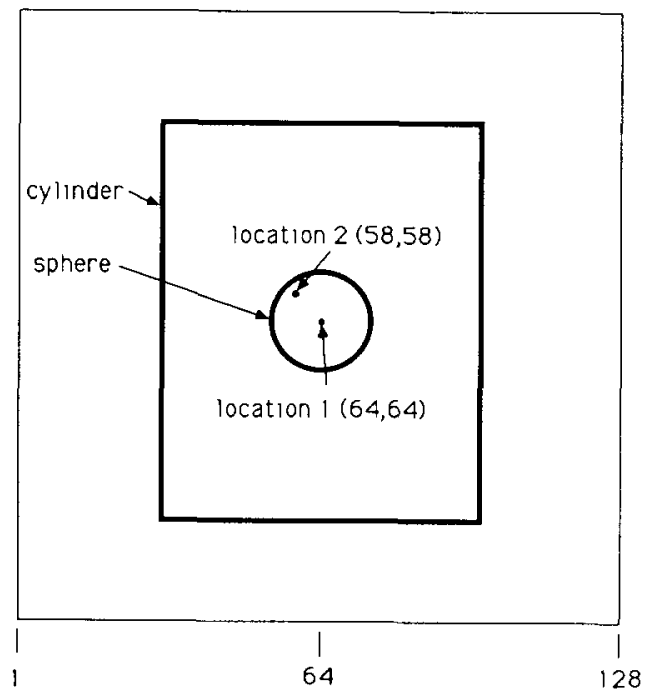

Fig. 1. A cross section of the phantom on top of the $128 \times 128$ image field. The two pixel locations used for testing are also shown.

with spectral fitting is also calculated. These tests are compared to ones run on experimental data previously measured with an Anger-camera as described in [2]. The geometry of the phantom is the same; the tested pixel location is the same as location 1; and the data are grouped 16 to 1 to approach no-noise statistics. The true direct counts in this case are obtained using the scatter fraction provided by Monte Carlo simulation and the total of measured counts in the $20 \%$ window.

For tests on noisy energy spectra, 100 independent noisy spectra are generated for each pixel-location, noise-level combination. The mean and standard deviation of the computed direct counts are calculated, as is the bias of the mean (the difference of the estimate from the true answer). Lastly, the average relative error, which reflects both the standard deviation and the bias, is computed. It is defined as

average relative error $=\frac{1}{100} \sum_{i=1}^{100} \frac{\left|\hat{c}_{t}-c\right|}{c}$,

where $\hat{c}_{t}$ is the computed direct counts for the $t$ th noisy energy spectrum and $c$ is the true direct counts.

\section{Results}

The tests on the ideal spectra were done with four different fitting window settings and four polynomial orders. The results in terms of the ratio of computed over true direct counts and of the percentage correction for the 16 different parameter combinations and two pixel locations are listed in table 1. Also listed in the table are the average of the corrected values and the 
Table 1

Test results on noise-free simulated data with different polynomial orders and fitting windows for locations 1 and 2

"Ratio" is the ratıo of the computed direct count to the true direct count, "percent correction" is the actual computed correction as a percentage of the required correction.

\begin{tabular}{|c|c|c|c|c|c|}
\hline \multirow{2}{*}{$\begin{array}{l}\text { Order } \\
\text { of } \\
\text { poly- } \\
\text { nomial }\end{array}$} & \multirow{2}{*}{$\begin{array}{l}\text { Fitting } \\
\text { window } \\
(\mathrm{keV})\end{array}$} & \multicolumn{2}{|c|}{ Location 1} & \multicolumn{2}{|c|}{ Location 2} \\
\hline & & Ratio & $\begin{array}{l}\text { Percent } \\
\text { correc- } \\
\text { tion }\end{array}$ & Rat1o & $\begin{array}{l}\text { Percent } \\
\text { correc- } \\
\text { tion }\end{array}$ \\
\hline 3 & $113-167$ & 1.088 & 63.7 & 1.093 & 82.8 \\
\hline 4 & $113-167$ & 1.067 & 72.7 & 1.024 & 95.5 \\
\hline 5 & $113-167$ & 1.066 & 72.9 & 1.031 & 94.3 \\
\hline 6 & $113-167$ & 1.057 & 76.5 & 0.962 & 107.0 \\
\hline 3 & $113-161$ & $1.114^{\mathrm{a})}$ & 53.5 & $1.156^{b)}$ & 71.3 \\
\hline 4 & $113-161$ & 1.011 & 95.3 & 0.889 & 120.0 \\
\hline 5 & $113-161$ & 1.104 & 57.5 & 1.104 & 80.9 \\
\hline 6 & $113-161$ & $1.008^{c)}$ & 96.9 & 0.848 & 128.0 \\
\hline 3 & $121-159$ & 1.071 & 708 & 1048 & 91.1 \\
\hline 4 & $121-159$ & 1.063 & 74.2 & 0952 & 109.0 \\
\hline 5 & $121-159$ & 1.061 & 75.0 & 0.962 & 107.0 \\
\hline 6 & $121-159$ & 1.094 & 61.7 & 0.886 & 121.0 \\
\hline 3 & $125-155$ & 1.068 & 724 & 1.014 & 974 \\
\hline 4 & $125-155$ & 1.077 & 684 & 0.893 & 119.0 \\
\hline 5 & $125-155$ & 1.072 & 70.8 & 0.914 & 116.0 \\
\hline 6 & $125-155$ & 1.161 & 34.3 & 1.118 & 78.3 \\
\hline \multicolumn{2}{|c|}{ Average } & 1.074 & 69.8 & 0.993 & 101 \\
\hline \multicolumn{2}{|c|}{ No correction } & 1.245 & 0.0 & 1.543 & 0.0 \\
\hline
\end{tabular}

a) Results for noisy data shown in fig. 3 .

b) Results for noisy data shown in fig. 4 .

c) Results for noisy data shown in figs. 2 and 3.
Table 2

Test results on experimental data with different polynomial orders and fitting windows The data were grouped 4 pixels by 4 pixels. The location is at the center of the sphere. "Rat1o" is the ratio of the computed direct count to the true direct count. The latter is determined from the Monte Carlo-determined scatter fraction and the total experimental counts within a $20 \%$ window. "Percent correction" is the actual computed correction as a percentage of the required correction.

\begin{tabular}{llcc}
\hline $\begin{array}{l}\text { Order of } \\
\text { polynomial }\end{array}$ & $\begin{array}{l}\text { Fitting } \\
\text { window }[\mathrm{keV}]\end{array}$ & Ratio & $\begin{array}{l}\text { Percent } \\
\text { correction }\end{array}$ \\
\hline 3 & $113-167$ & 1.054 & 78.0 \\
4 & $113-167$ & 1.088 & 64.3 \\
5 & $113-167$ & 1.008 & 97.0 \\
6 & $113-167$ & 1.061 & 75.2 \\
3 & $113-161$ & 1.074 & 69.8 \\
4 & $113-161$ & 0.996 & 101 \\
5 & $113-161$ & 1.054 & 78.0 \\
6 & $113-161$ & 0.961 & 116 \\
3 & $121-159$ & 1.025 & 90.1 \\
4 & $121-159$ & 1.035 & 86.0 \\
5 & $121-159$ & 0.992 & 103 \\
6 & $121-159$ & 1.041 & 83.4 \\
3 & $125-155$ & 1.009 & 96.4 \\
4 & $125-155$ & 1.052 & 78.8 \\
5 & $125-155$ & 1.039 & 84.1 \\
6 & $125-155$ & 0.981 & 107 \\
Average & & 1.029 & 88.1 \\
No correction & & 1246 & 0.0 \\
\hline
\end{tabular}

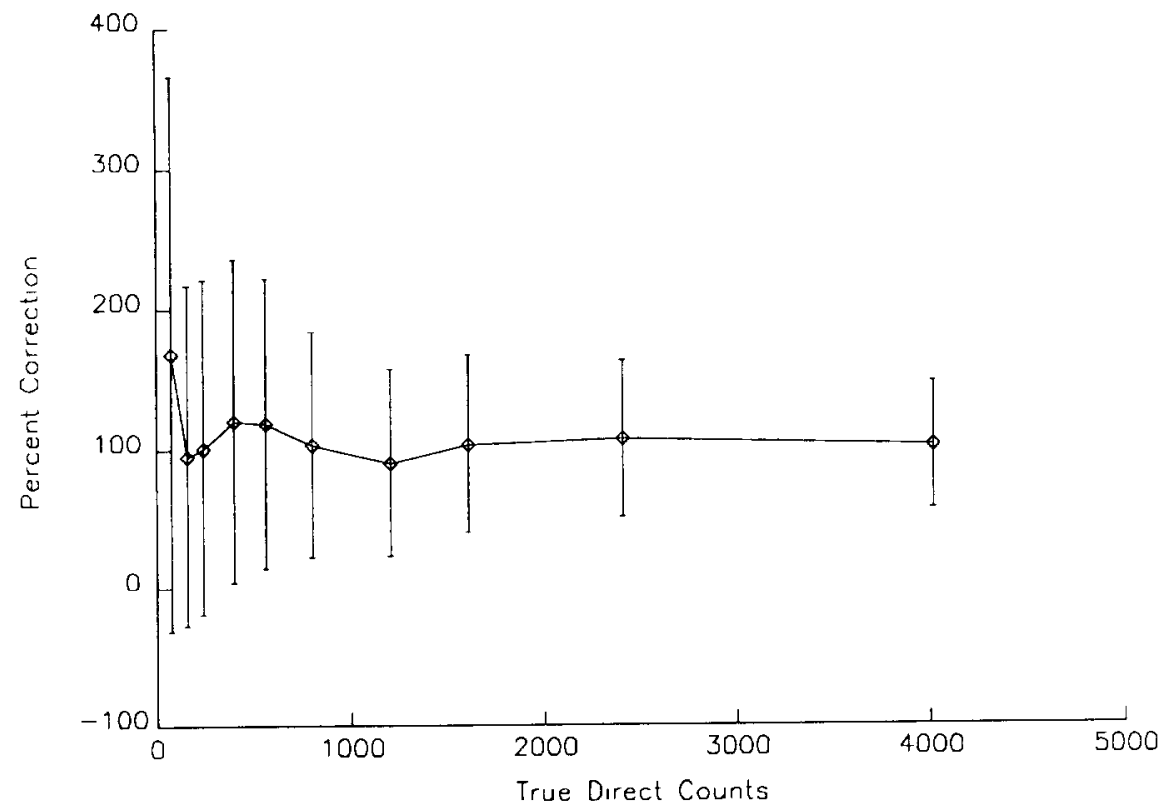

Fig. 2. The mean percent correction for noisy data versus true direct counts with the standard deviation plotted as error bars for location 1. Fitung parameters are: 6th-order polynomial and fitting from 113 to $161 \mathrm{keV}$. 


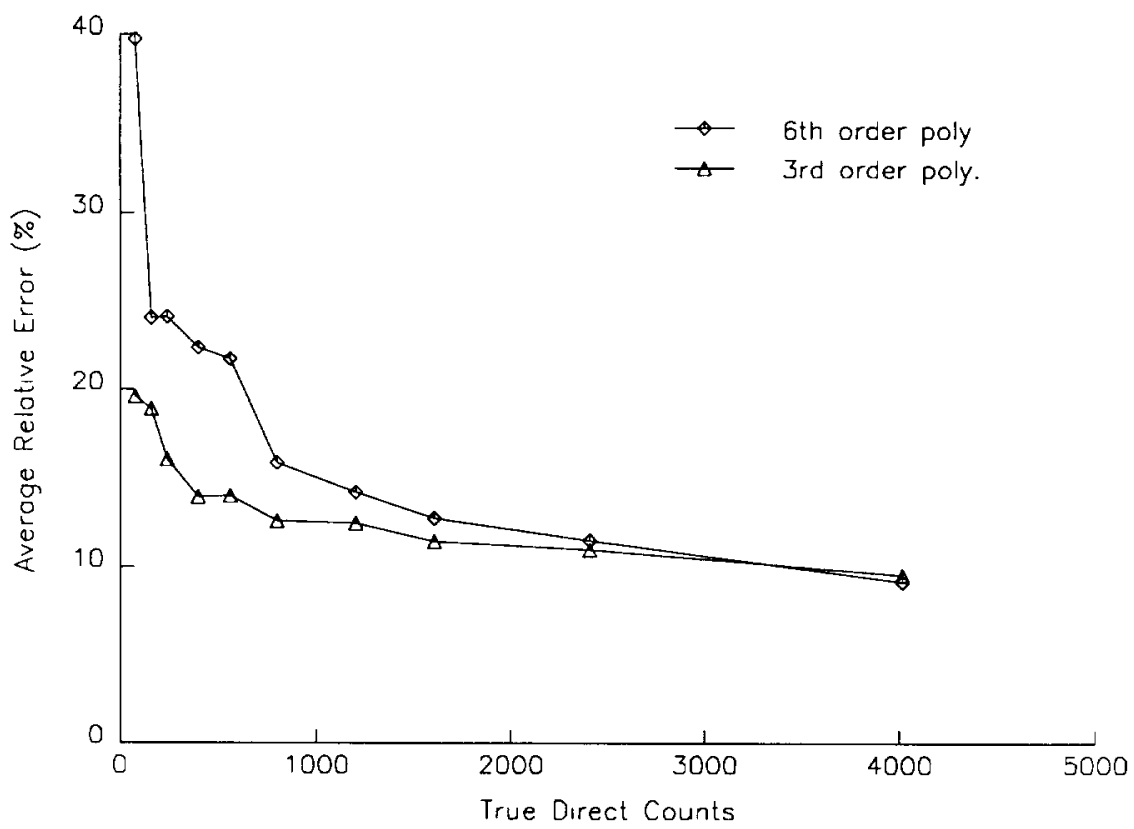

Fig. 3. The average relative error of computed direct counts for noisy data plotted versus true direct counts for location 1 with two different polynomial orders. Fitting window is from 113 to $161 \mathrm{keV}$. This data is the same as that for which the standard deviation and bias are given in table 3 .

values without correction. The calculated-to-true ratio for direct counts ranges from 0.848 to 1.16 over all the cases meaning that the end result is reasonably good, no matter what fitting parameters are chosen.
Trying to optimize the correction is a more difficult proposition. For example, the percent of the needed correction which is accomplished is different for the two locations. For location 1 , where there is relatively less

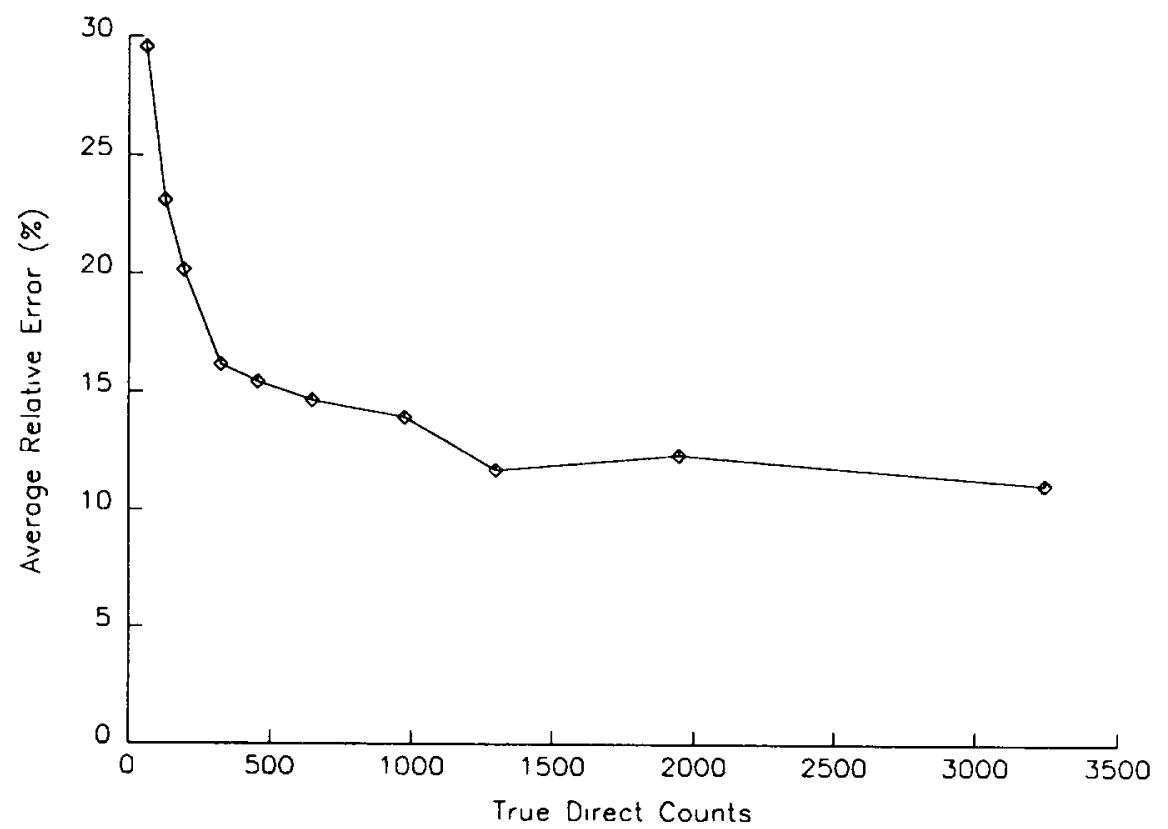

Fig. 4. The relative error of computed direct counts for noisy data plotted versus true direct counts for location 2 with 3 rd-order polynomial. Fitting window is again from 113 to $161 \mathrm{keV}$. 
scattering, the algorithm underestimates the scattering with all the tested settings. The average percent correction is about $70 \%$, with the best case being almost $97 \%$ and the worst case $34.3 \%$. For location 2, the algorithm overestimates the correction in half of the 16 cases, and underestimates it in the other half. The average percent correction is about $101 \%$, the best case being $97.4 \%$ and the worst $128 \%$. In addition, the best parameter combination for location 1 is the worst for location 2 .

Table 2 contains the same tests for the experimentally measured data. The single experimental result shows a better correction than the noise-free Monte Carlo for location 1 but one not as good as the Monte Carlo for location 2. The calculated-to-true ratio ranges from 0.981 to 1.08 , while the average percent correction is $88.1 \%$ with the best case being $101 \%$ and the worst case $69.8 \%$.

All the tests on the noisy energy spectra were done with a fitting window from 113 to $161 \mathrm{keV}$. For location 1 , fig. 2 shows the mean correction obtained and the standard deviation of that correction, versus true direct counts with a 6 th-order polynomial. In this case, the mean correction stays at the ideal $100 \%$ all the way down to the next-to-lowest true direct count. The standard deviation, however, increases by a factor of 2.6 . For another case, the mean correction deteriorated as the true direct count decreased and the standard deviation worsened, but not quite as much. Generally, the behavior seems to vary with the case and the shape of the curve is somewhat irregular with only the 100 realizations measured.

Listed in table 3 are the bias and standard deviation with different noise levels and two different polynomial orders at location 1. Comparing the bias and the standard deviation at the same noise level, a trade-off is

Table 3

Bias and standard deviation of the average computed direct counts for noisy spectra for 3rd- and 6th-order polynomials at location 1 , the fitting window is from 113 to $161 \mathrm{keV}$ in both cases

\begin{tabular}{|c|c|c|c|c|}
\hline \multirow{2}{*}{$\begin{array}{l}\text { True } \\
\text { direct } \\
\text { counts }\end{array}$} & \multicolumn{2}{|c|}{ 3rd-order polynomial } & \multicolumn{2}{|c|}{ 6th-order polynomial } \\
\hline & B1as [\%] & $\begin{array}{l}\text { Relative } \\
\text { std. dev. [\%] }\end{array}$ & $\operatorname{Bias}[\%]$ & $\begin{array}{l}\text { Relatıve } \\
\text { std. dev. [\%] }\end{array}$ \\
\hline 80 & 16.6 & 15.1 & -16.6 & 49.0 \\
\hline 161 & 18.2 & 10.2 & 1.3 & 29.9 \\
\hline 241 & 15.5 & 9.42 & -0.2 & 29.5 \\
\hline 402 & 13.5 & 7.39 & -4.8 & 28.4 \\
\hline 562 & 13.9 & 6.76 & -4.4 & 25.5 \\
\hline 803 & 12.4 & 5.90 & -0.8 & 19.8 \\
\hline 1205 & 12.4 & 4.90 & 24 & 16.5 \\
\hline 1606 & 114 & 4.19 & -0.8 & 15.6 \\
\hline 2410 & 11.0 & 3.13 & -1.7 & 13.6 \\
\hline 4016 & 93 & 4.90 & -0.5 & 11.1 \\
\hline
\end{tabular}

observed in nine of ten cases. That is, with the high-order polynomial, the bias is low but the standard deviation is high; the reverse is true for the low-order one.

The average relative error for noisy energy spectra is shown in figs. 3 and 4. For location 1 (fig. 3) two different orders of polynomial - cubic and 6th-order were tested. At low count levels the cubic fit gives better results, while at high count levels, the two curves merge. For location 2 only a cubic polynomial was tested and the curve shape is similar. In all three cases, when the count level is higher than 700 , the fitting method provides a value for direct counts accurate within $20 \%$ or better on average.

\section{Discussion}

Determining the parameters of the spectral-fitting method is important for its effective application. Overall, our results show that the method is effective. Compared with no correction at all, test cases on noise-free spectra showed significant improvement with all parameter settings for both the simulated and experimental data. On the other hand, the simulated results suggest that the best choice of parameters depends on the spatial location. This dependency occurs because the shape of the scattered spectrum changes with the change of spatial location. Optimization of order and window may depend on the object in which one is most interested and on the noise level which is expected. For quantification of a hot tumor in a cold background at a given noise level, one could look for the least error in total direct counts over the ROI of the sphere. Whether this error would vary sharply with the parameters (and yield a good advantage from optımization) or not remains to be seen.

As far as noise, the present method is basically robust. It yields an average relative error of $10 \%$ for the direct counts with good statistics for each of the three tests run. This error remains low (less than $20 \%$ ) until the number of true direct counts drops below 700. The choice of the polynomial order appears to control the trade-off between bias and variance of the results for the location and the window tested. An even more robust correction scheme which would be less sensitive to noise might involve a different form for the scatter component.

\section{Acknowledgements}

This work was supported by PHS Grant numbers R01 CA38790, R01 CA32846 and P0 CA42768 awarded by the National Cancer Institute, DHHS. 


\section{References}

[1] K.F. Koral, X. Wang, W.L. Rogers, N.H. Clinthorne and X. Wang, J. Nucl. Med. 29 (1988) 195.

[2] K.F. Koral, X. Wang, K.R. Zasadny, N.H. Clinthorne,
W.L. Rogers, C.E. Floyd Jr. and R.J. Jaszczak, Phys. Med. Biol., in press.

[3] C.E. Floyd, R.J. Jaszczak. C.C. Harris and R.E. Coleman, Phys. Med. Biol. 29 (1984) 1217. 